\title{
Scale-free behavioral dynamics directly linked with scale-free cortical dynamics
}

\author{
Sabrina A. Jones ${ }^{1}$, Jacob H. Barfield ${ }^{1}$, Woodrow L. Shew ${ }^{1 *}$ \\ ${ }^{1}$ Department of Physics, Integrative Systems Neuroscience Group, University of Arkansas,
} Fayetteville, AR, US, 72701

*Corresponding author. Email: shew@uark.edu

\begin{abstract}
Naturally occurring body movements and collective neural activity both exhibit complex dynamics, often with scale-free, fractal spatiotemporal structure, thought to confer functional benefits to the organism. Despite their similarities, scale-free brain activity and scalefree behavior have been studied separately, without a unified explanation. Here we show that scale-free dynamics of behavior and certain subsets of cortical neurons are one-to-one related. Surprisingly, the scale-free neural subsets exhibit stochastic winner-take-all competition with other neural subsets, inconsistent with prevailing theory of scale-free neural systems. We develop a computational model which accounts for known cell-type-specific circuit structure and explains our findings. Our results establish neural underpinnings of scale-free behavior and clear behavioral relevance of scale-free neural activity, which was previously thought to represent background noise in cerebral cortex.
\end{abstract}

One-Sentence Summary: Winner-take-all competition among cortical neurons underpins shared fractal spatiotemporal dynamics of behavior and brain. 
From fidgeting to expeditions, natural body movements manifest over a very broad range of spatiotemporal scales. The complexity of such movements is often organized with fractal structure, scale-free fluctuations spanning multiple spatiotemporal orders of magnitude (1-4). Such behavioral complexity may be beneficial for foraging (3-6), visual search (4), decision making based on priority $(7,8)$, flexible switching of behavior $(9)$, and perhaps more. Similarly, fluctuations of ongoing neural activity in cerebral cortex can exhibit fractal, scale-free fluctuations like the spatiotemporal cascades sometimes referred to as 'neuronal avalanches' (10-18) and long-range temporal correlations (19-21). Considering these two observations together, a simple, yet unanswered question arises. Are scale-free dynamics in cerebral cortex related to scale-free body movements? Or are these statistical similarities merely superficial, without any direct link?

According to the prevailing view, scale-free ongoing neural activity is interpreted as "background" activity, not directly linked to behavior. Indeed, ongoing activity is typically defined as the activity that persists even when an organism is at rest, not engaged by any particular motor task or sensory input: the organism is "doing nothing" while the ongoing activity continues to "do something." Further support for this view comes from in vitro slice studies and computational models, which indisputably show that scale-free ongoing dynamics of neuronal networks can be truly autonomous, occurring without interactions between the network and the outside world $(10,22-24)$. However, the fact that ongoing activity can occur autonomously does not necessarily mean that it always does so. Indeed, animals are not really "doing nothing" during periods of wakeful rest between controlled tasks or sensory stimuli. In head-fixed mice, for instance, the animals often run on a wheel, move their whiskers, move their eyes, change their vigilance state (as indexed by pupilometry), and change their body posture and muscular tone. Previous studies of scale-free ongoing cortical dynamics have not accounted for such ongoing body movements. And previous studies of scale-free behavior have not measured brain activity. Moreover, no previous study relating behavior to ongoing brain activity has accounted for the scale-free structure of neural and behavioral fluctuations. Therefore, there remains a possibility that scale-free neural activity is comprised of neural events that are directly related to specific behavioral events. If this is the case, it would dramatically revise the typical interpretation of scale-free ongoing activity and provide direct evidence that scale-free behavior and scale-free brain activity are inseparably related. Here we test this possibility in awake headfixed mice.

\section{Results}

Our results are based on simultaneous recordings of more than 10000 pyramidal neurons in visual cortex of awake mice ( 7 mice, 9 recordings, Fig S1), performed and first reported by Stringer et al (25). These recordings were obtained using multiplane Ca2+ imaging of a slow calcium indicator. In addition to the neural activity, several aspects of behavior and body movements were also recorded. Here, we focused on four aspects of behavior. First, we studied run speed, which was assessed using an optical sensor and a floating spherical treadmill. Second, we examined pupil diameter, which was obtained from a camera targeting the faces of the mice. Pupil diameter dynamics are associated with changes in arousal and other aspects of body movement and sensory input. Third, we studied changes in direction of gaze by tracking the speed of the center of the pupil as the mice looked around. Finally, we used previously developed methods to study whisker motion (25), which was also obtained from the face camera. 

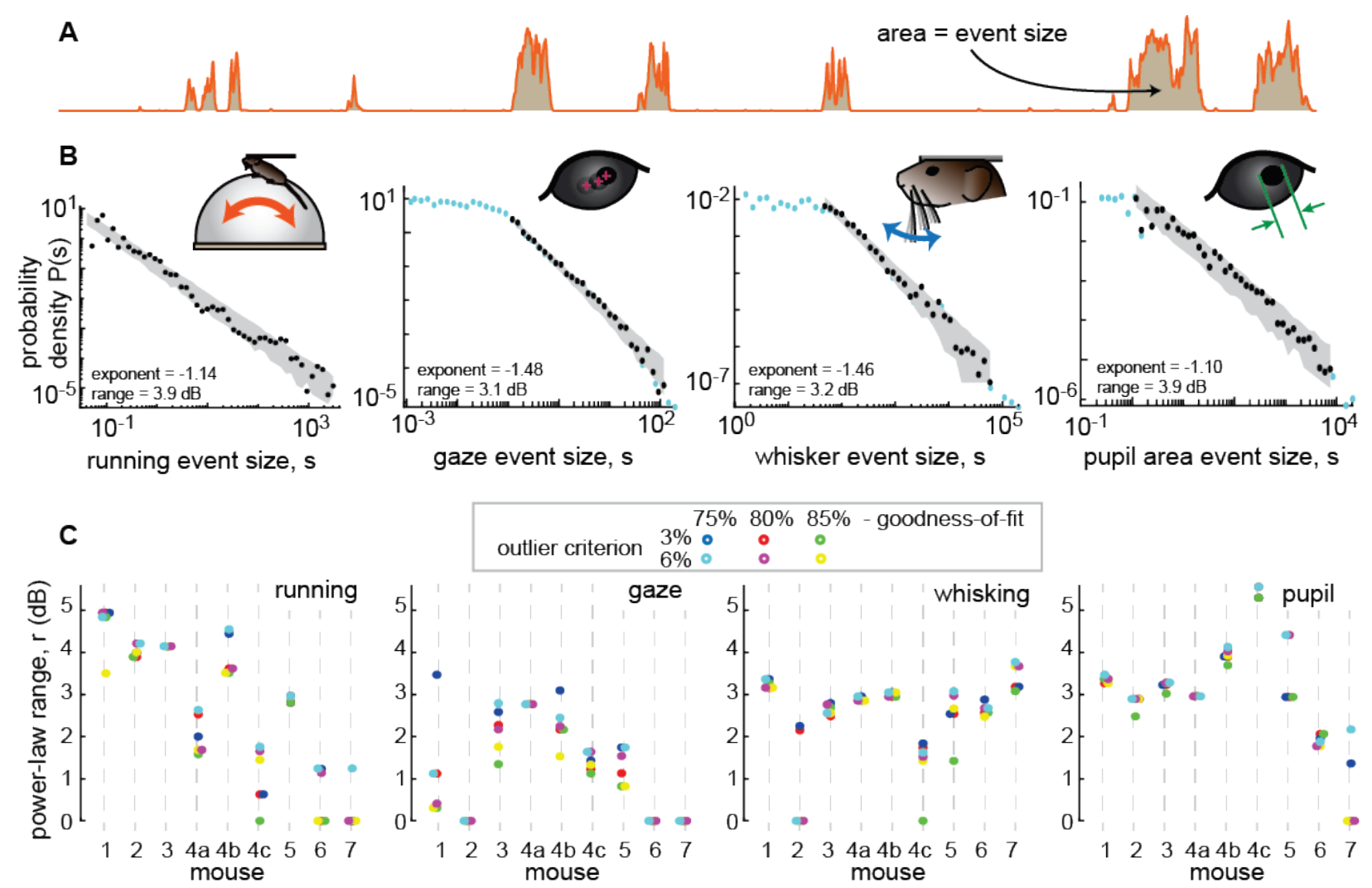

Figure 1. Spontaneous behavior is often scale-free. A) Behavioral events (run speed, in this example) are defined by excursions above the median; event size is the area (shaded patch) between the curve and the median. B) Four examples of behavioral event size distributions with large power-law range $r>3 \mathrm{~dB}$. Black points indicate the part of the distribution that is fit well by a power law. Blue points fall outside the power-law range. Gray patch indicates the expected variability (10th to 90th percentile) of the best-fit power law. C) Summary of power-law range for all mice and all behaviors. Gaze dynamics were least likely to have a large power-law range. All mice had at least one behavior with a very large $(>3 \mathrm{~dB})$ power law range. Changes in power-law fitting parameters (goodness-of-fit criteria and outlier criteria) did not change our general conclusions.

\section{Scale-free behavior}

The behavior tended to occur in bursts; rest periods were punctuated with well-defined bouts of body movement. All four of the behavioral variables we studied tended to start and stop together, approximately, but differed in their more detailed, faster fluctuations (Fig S1). We first sought to determine whether the behaviors exhibited scale-free dynamics. To this end, we first defined each bout of elevated body activity, hereafter called a behavioral 'event,' based on a threshold (the median of the entire time series). Each behavioral event began when the behavioral time series, run speed for example (Fig 1A), exceeded the threshold and ended when it returned below the threshold. The 'size' of each behavioral event was defined as the area between the threshold and the variable during the event (Fig 1A). This definition of events and their sizes is motivated by previous studies of scale-free neural activity (26), typically referred to as neuronal avalanches. In the case of run speed, the event size corresponds to an effective distance traveled during the event. If the animal behavior is scale-free, one expects the distribution of behavioral event sizes to have a power-law form. We found that these distributions often were in the form of a power-law over a wide range of event sizes (Fig 1B). For example, the run speed event size distribution for mouse 1 was scale-free for nearly 4 orders of magnitude of event sizes (power-law range, $r=3.9 \mathrm{~dB}$ ). To rigorously assess the range over which the data is power-law distributed, we used a maximum likelihood fitting algorithm that 
accounts for the number of events observed and the possibility of a cutoff at the head and tail of the distribution (Methods). Our approach builds on that used in previous studies (11). We report the power-law range $\mathrm{r}$ in $\mathrm{dB}$, i.e. the number of orders of magnitude. If no range meets our fit criteria for statistical significance, we report $r=0$.

Although not all behaviors were scale-free over such a large range for all recordings, we conclude that in these experiments, most of the mice exhibited scale free behaviors, particularly for running and pupil fluctuations (Fig 1C). These conclusions were robust to changes in the two most important parameters for the power-law fitting algorithm: the goodness-of-fit criterion and the outlier exclusion criterion.
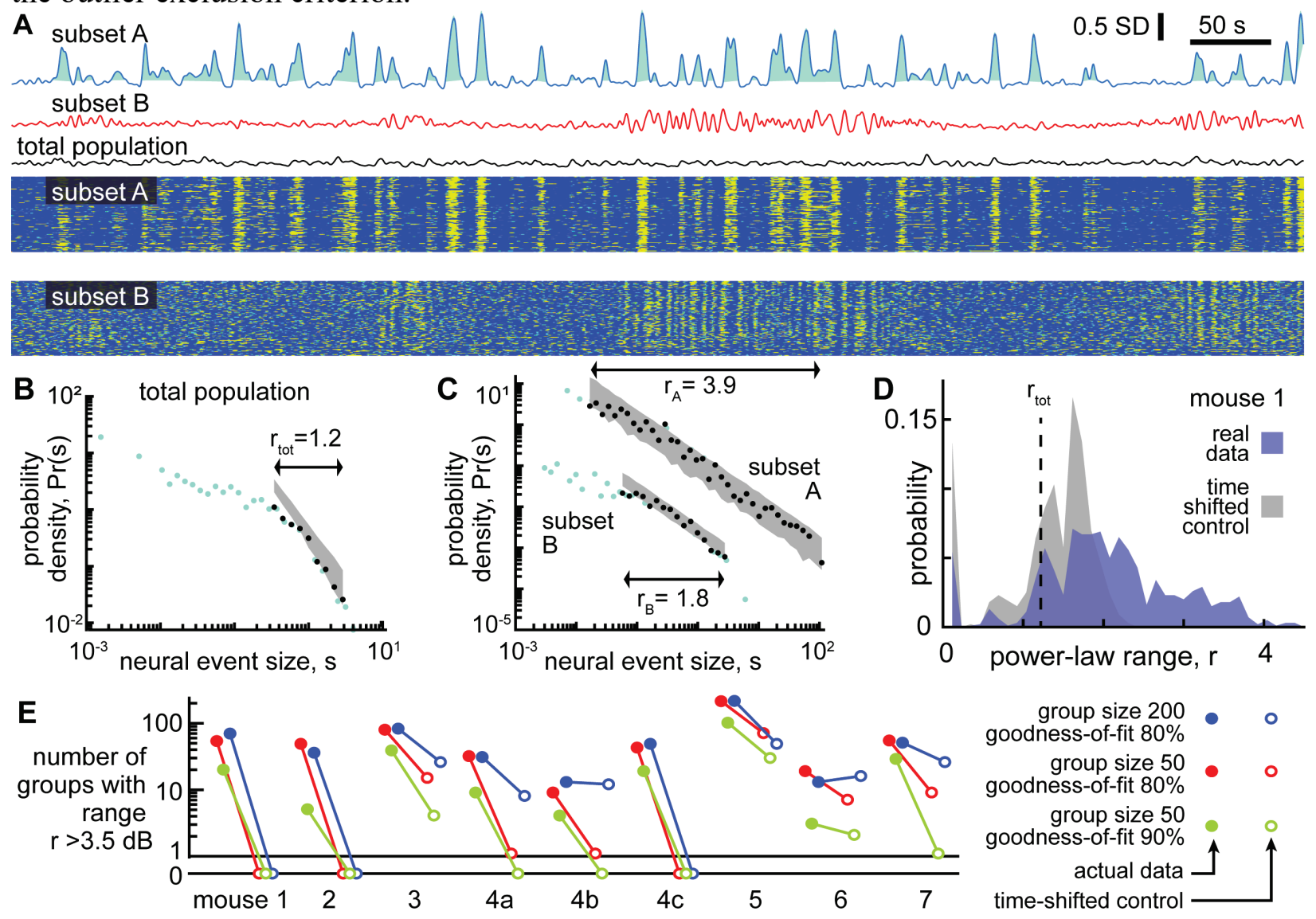

Figure 2. Certain subsets of neurons exhibit scale-free dynamics, but total population does not. A) Activity of the total neural population (black) and two subsets of neurons (blue, red) are shown. Each excursion above the median is one neural event (blue shaded area for subset A). Examples in panels A, B, and C are from mouse 1. B) For the total population, the distribution of neural event sizes is not well fit by a power law (small range $\mathrm{r}_{\mathrm{tot}}=1.2$ $d B)$. C) Certain subsets of neurons exhibited very large power-law range $\left(r_{A}=3.9 \mathrm{~dB}\right)$, while other subsets did not $\left(r_{B}=1.8 \mathrm{~dB}\right)$. D) Distribution of power-law range $r$ for 1000 subsets from mouse 1 (blue). Power law range $r$ reached $4.5 \mathrm{~dB}$ for some subsets, but did not exceed $2.6 \mathrm{~dB}$ for time-shifted controls (gray) in this mouse. E) Summary of all mice, showing the number of subsets (out of 1000) with power-law range $r>3.5 \mathrm{~dB}$, compared to time-shifted controls. Results were qualitatively unchanged for different group size or goodness-of-fit criteria.

\section{Scale-free neural activity}

Next, we turned to the neural activity to test whether it was scale-free like the behavior. First, we averaged over all neurons to obtain a single population activity time series. Then, we treated the neural data in a similar way to the behavioral data. We defined neural events with a median threshold and defined event size as the area between the threshold and the data during the event 
(Fig 2A). The resulting distributions of neural event sizes were not scale-free; they did not manifest as a straight line on a log-log plot and were poorly fit by a power-law distribution (Fig 2B). For all 9 recordings, we found that $r=1.5 \pm 0.9 \mathrm{~dB}$ (mean $\pm \mathrm{SD}$ ). Such a short power-law range should be interpreted as evidence against any power-law at all, because any function can be fit on a short range of data.

This lack of scale-free dynamics for the neural population was surprising considering two facts together: first, the behavior exhibited scale-free dynamics (Fig 2), and second, many neurons are strongly correlated with behavior (Fig S1 and (25)). One possible explanation of this puzzling observation is that considering the entire population together obscures the scale-free dynamics of certain subsets of neurons. Next, we set out to test this possibility. Since we did not know, a priori, which neurons might be in these subsets, we adopted a brute force, shotgun search. First, we picked a 'seed neuron' at random from the entire population. Then we identified the 50 neurons that were most correlated with the seed neuron (we also tried 100 or 200 neurons without major impacts on the following results). We averaged the activity of these 50 neurons to obtain a single time series and proceeded to define events and examine their size distribution. Two examples of time series obtained from subsets of neurons in this way are shown in Fig 2A. We repeated this process for 1000 different seed neurons. We found that some subsets of neurons were indeed scale-free, with power-law scaling over more than 4 orders of magnitude, while other subsets were not scale-free (Fig 2C).

With so many neurons to choose from and a shotgun approach like this, it is important to avoid chance-level false-positive conclusions. As a conservative control for this possibility, we repeated the analysis, but with surrogate versions of each neuron's activity, generated by applying a random time shift relative to other neurons. This time-shifted control data, thus, has identical statistics to the real data at the single neuron level, but correlations among neurons are abolished, on average. For time-shifted controls, we found that large power-law ranges (greater than $3.5 \mathrm{~dB}$ ) were rare and not found at all in the majority of recordings (Fig 2D,E). This conclusion was robust, largely unchanged for different choices of group size and goodness-of-fit criteria used for the power-law fitting (Fig 2E).

\section{Linking scale-free brain and behavior}

Having established that certain subsets of neurons have scale-free dynamics and that behavior has scale-free dynamics, we next sought to assess how the neural and behavioral dynamics are related. We first computed the correlation coefficient between each behavior time series and each neural subset time series. The correlation was very weak between the total neural population time series and behavior (red x's in Fig 3). For the 50-neuron subsets, we found a very wide range of behavioral correlation values, from 1 to -1 (Fig 3). Interestingly, the subsets with the greatest correlation with behavior also tended to have the widest ranges of power-law scaling. Moreover, the neural subsets with the smallest power-law range were most often near zero correlation with behavior. These results strongly suggest that the scale-free subsets of neurons are directly related to scale-free behavior. It is also interesting to note that many of the subsets that were strongly anticorrelated with behavior also had large power-law range (the Ushaped relationships in Fig 3).

The calculation of correlation coefficients, like those in Fig 3, reflect an average over time, leaving open several questions. Are the high correlations in Fig 3 simply due to similar coarse, on-off dynamics of the neural and behavioral activity, or are there strong correlations at the more 

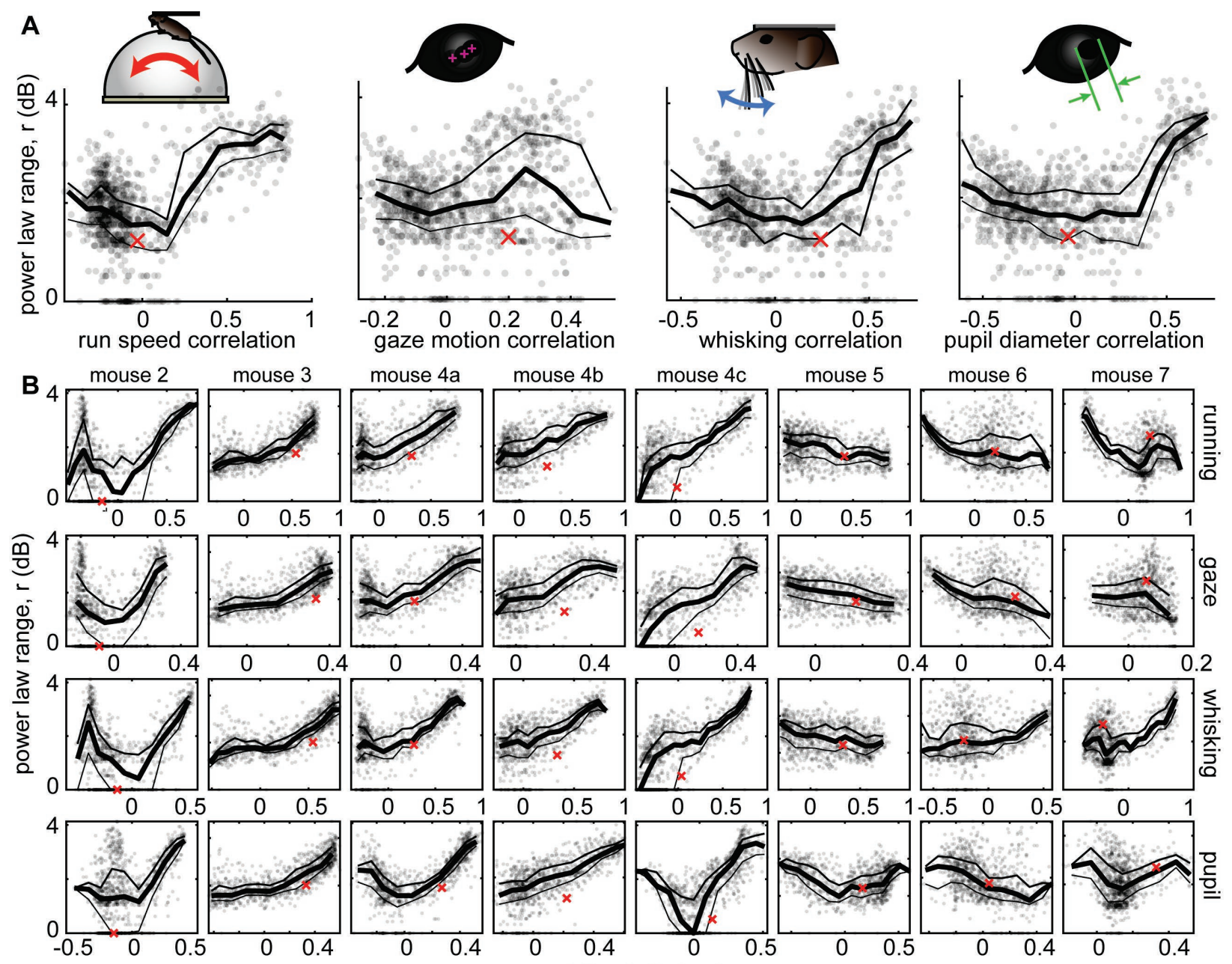

correlation to behavior

Figure 3. Scale-free neural subsets correlate with behavior, but non-scale-free subsets do not. A) Each point represents the power-law range $r$ and behavioral correlation of one neural subset from mouse 1. Subsets with large $r$ tend to be most correlated with behavior. Thick line is a moving average of points; thin lines delineate quartiles. The red $\mathrm{x}$ represents the total population. B) Same as panel A for the other 8 recordings.

detailed level of the faster fluctuations during a single behavioral event? Are all behavioral events equally correlated to their concurrent neural events, or are certain neural events from certain subsets of neurons more strongly related to behavioral events? To answer these questions, we computed event-specific correlations - computed between the behavior time series and the neural subset time series during each behavioral event, for each neural subset (Fig 4A). For instance, for 1000 neural subsets and 1000 behavioral events (which are typical numbers for this data), we would calculate 1 million correlation values. Each correlation is based on the relatively short time series between the start and stop of the corresponding behavioral event. These event-specific correlations were often quite high $(>0.9)$. However, the chance-level occurrence of such high correlations can also be high for such short time series. We account for such chance-level correlations by repeating the calculations for time-shifted control data (Methods), which defines the event-specific, subset-specific chance-level occurrence rate.

First, we asked how many neural subsets were strongly correlated with each behavioral event and whether this count depended on behavioral event size. We found that many behavioral events had a significant number of such strongly correlated subsets and that the larger behavioral events tended to have more strongly correlated subsets (Fig 4B). Next, we asked how many 
behavioral events were strongly correlated with each neural subset and whether this count depended on the power-law range of the neural subset. We found that many subsets were strongly correlated with a significant number of behavioral events and that the subsets with greater power-law range tended to be correlated with more behavioral events (Fig 4C). The example results in Fig 4 are shown for all mice and all types of behaviors in Figs S2 and S3. Finally, we considered the time during which the mouse was active and asked what fraction of that time occurred with a significant number of strongly correlated neural subsets. This fraction of time was often as high as 0.5 (Fig 4D). Thus, we conclude that the detailed, fast fluctuations of behavior are significantly and strongly correlated with scale-free neural activity.

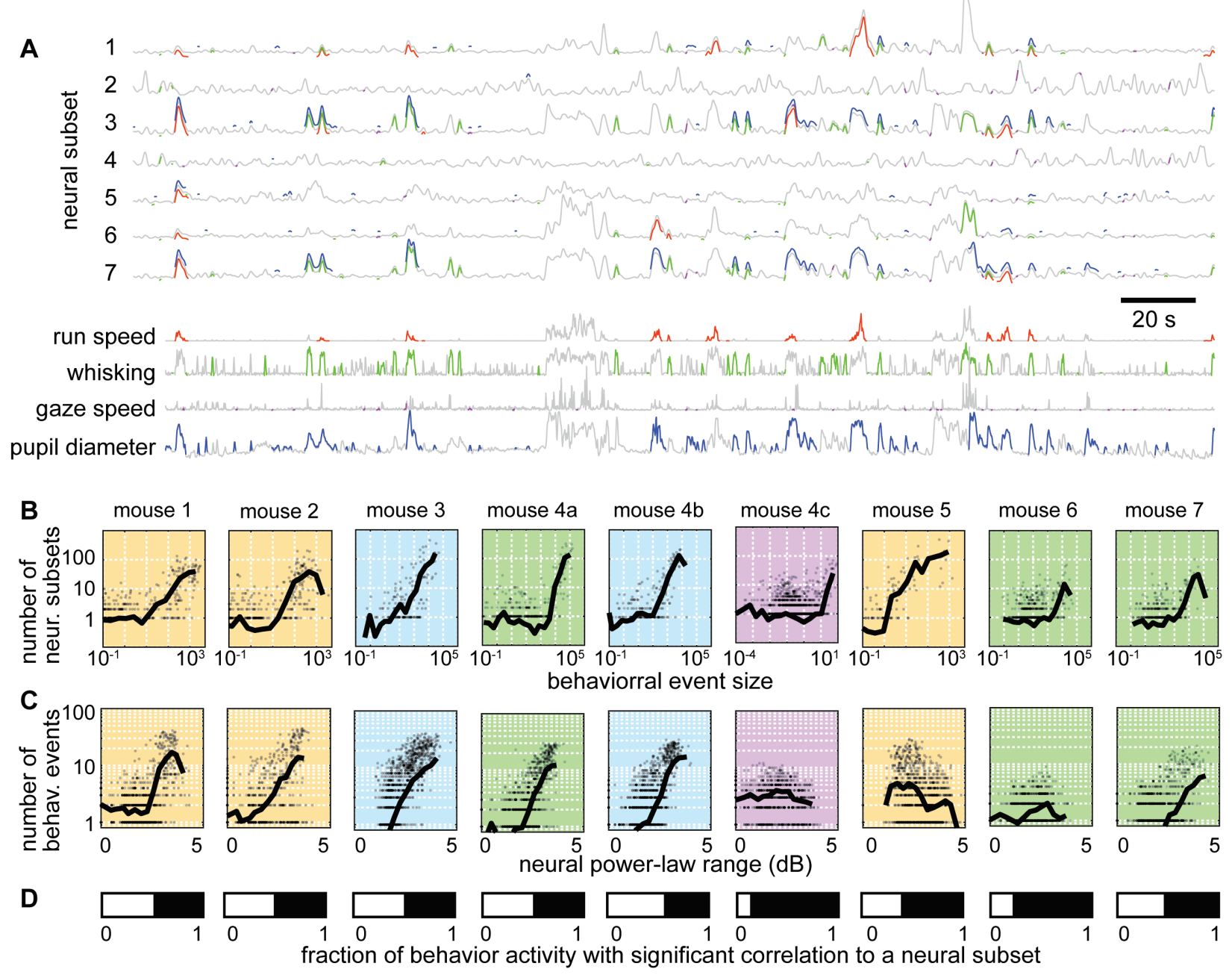

Fig 4. Individual neural events correlate with specific behavioral events. A) Time series from 7 example neural subsets and the four behaviors. Each behavioral event with strong correlation is indicated with a pair of colored line segments, one on the behavioral time series, one on the neural time series. B) Neural events with greater size were strongly correlated with more neural subsets. C) Neural subsets with greater power-law range were typically strongly correlated with a larger number of behavioral events. For panels $\mathrm{B}$ and $\mathrm{C}$, each plot summarizes one recording, with one gray point per behavioral event. Black line is a moving average of the points. Background color indicates type of behavior: orange - running; blue - pupil; green - whisking; purple - gaze. D) The white bar indicates the fraction of active time with strong correlation to a significant number of neural groups. 

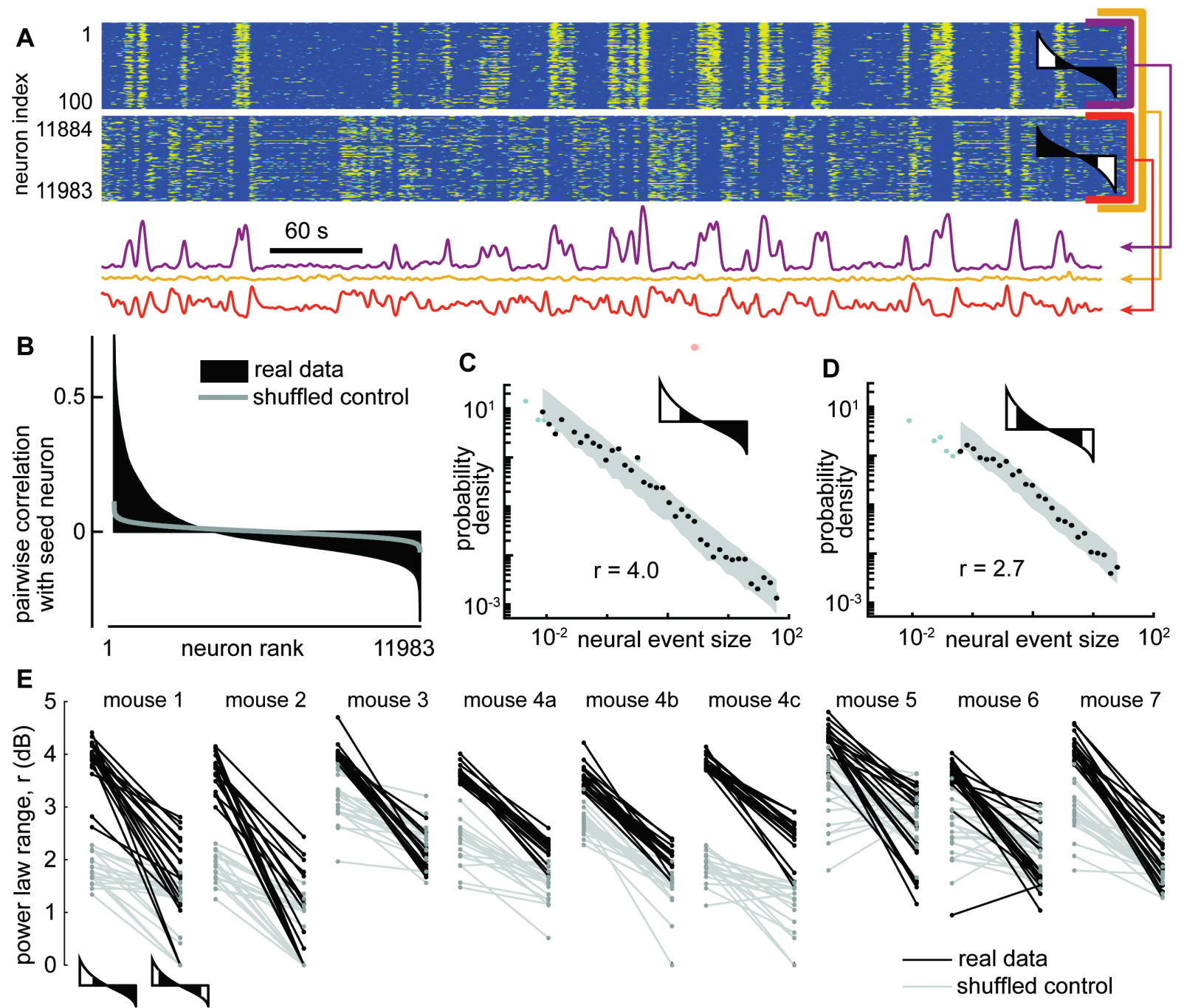

Fig 5. Cancelation between anticorrelated neural subsets hide scale-free neural activity. A) Activity of a subset of 100 neurons (top, purple) with large power-law range, another subset of 100 neurons (bottom, orange) that are anticorrelated with first subset, and the total population (yellow). B) Example correlation spectrum, showing the pairwise correlation coefficients between one seed neuron and all other neurons, ranked in descending order. Gray control spectrum based on randomly time-shifted neurons. C) Example neural event size distribution for a subset based on the top 100 most correlated neurons. D) Example event size distribution for a subset based on the top 50 most correlated neurons and 50 most anticorrelated neurons. Note that the power-law range is greatly reduced compared to panel C. E) The power-law range for the 50 most correlated (left) is much greater than that of the 50 most extreme (right) neurons. Shown are results for the 20 subsets with the greatest power law range for each mouse. Gray lines represent time-shifted controls, which exhibit smaller power-law range and smaller drop in range due to anticorrelated neurons. In panels A, C, D, and E, the cartoon correlation spectra indicate which neurons were included (white) in subsets and which were not (black).

\section{Anticorrelated neural subsets}

How is it possible for the total population to not exhibit scale-free fluctuations, while certain subsets are scale-free? A clue to this mystery is apparent upon close inspection of the results in Fig 3. Some neural subsets are strongly correlated with behavior, while other subsets are strongly anticorrelated, similar to previous reports from motor cortex (27) and prefrontal cortex (28). Since the scale-free subsets are often strongly correlated with behavior, it stands to reason that some neural subsets are strongly anticorrelated with the scale-free subsets. We show in Fig 5 that this fact explains why the total population does not exhibit scale-free dynamics. When 
two anticorrelated subsets are averaged together, they cancel out, resulting in relatively small fluctuations at the level of the total population (Fig 5A). The broad range of correlations and anticorrelations in each recording are more apparent using 'correlation spectra' (Fig 5B). For each previously defined seed neuron, we ranked all the other neurons according to their correlation with the seed neuron. These correlation spectra often reveal large numbers of strongly anticorrelated neurons. To directly show how cancelation of anticorrelated subsets abolishes scale-free dynamics, we re-computed the power-law range, but this time defined each subset to include the 25 most correlated and the 25 most anti-correlated neurons, relative to each seed neuron. The power-law range for these canceling subsets was greatly reduced compared to the original correlated subsets for all recordings (Fig 5C-E).

\section{Stochastic winner-take-all competition and criticality}

What mechanisms might be responsible for scale-free dynamics among certain subset of neurons that are obscured due to cancelation with anticorrelated subsets of neurons? Theoretical models for studying scale-free dynamics in neural networks have often employed randomly connected (Erdos-Renyi), networks of excitatory and inhibitory neurons, tuned to operate at criticality (22, $23,26)$. However, such models do not manifest qualitatively different slow fluctuations in different subsets of neurons. More specifically, there are no significant anticorrelations among subsets. How can traditional models be changed to account for our observations? Here we propose that a non-random network structure is needed. Indeed, experiments have shown that connectivity among cortical neurons is far from random (29-31); this non-random structure is particularly pronounced among different types of inhibitory neurons (31-34). To our knowledge, this cell-type-specific connectivity has been ignored in previous theoretical accounts of scale-free neural activity.

Which aspects of this network structure might explain our findings? We initially sought clues from well-known circuit motifs that generate the anticorrelated activity underlying many types of animal locomotion (35). A common motif in these circuits, sometimes termed 'crossing inhibition' or 'winner-take-all,' entails two excitatory circuit nodes, say e+ and e-, whose activity is anticorrelated because they interact via at least one inhibitory node; e+ excites the inhibitory node, which suppresses e-, and vice versa, e- excites the inhibitory node which suppresses e+ (Fig 6A-D). Such circuit motifs certainly are common in cortex, but they are mixed and interconnected with numerous other motifs. Even Erdos-Renyi networks contain many motifs of this type. Is it plausible that 'winner-take-all' motifs are responsible for our findings?

As shown in Fig 6A, we tested this possibility using a computational model of 1000 binary neurons divided into two excitatory groups (e+ and e- with 400 neurons each) and two groups of inhibitory neurons (i+ and $i-$ with 100 neurons each). We considered two inhibitory groups, instead of just one, to account for previous reports of anticorrelations between VIP and SOM inhibitory neurons in addition to anticorrelations between groups of excitatory neurons (28). The excitatory groups were densely connected within each group ( $50 \%$ connectivity) and sparsely connected across groups (5\%), consistent with experimental observations $(29,30)$. We also included dense crossing inhibition and crossing disinhibition according to known connectivity among some inhibitory cell types (31-34). Excitatory and inhibitory synaptic weights were of the same absolute magnitude, but opposite in sign, and the connectivity matrix was normalized such that its largest eigenvalue was 1 (Fig 6B), following previous studies of scale-free dynamics and criticality $(23,26,36)$. Each neuron fired probabilistically, in proportion to the sum of its inputs (Methods). 
A
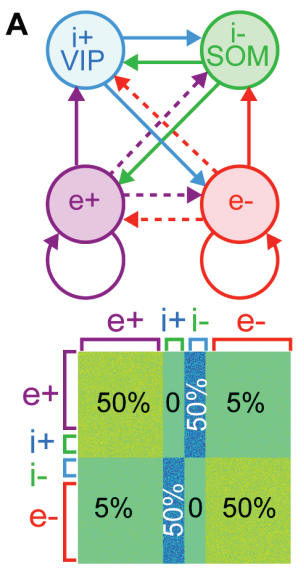

$B \operatorname{Im}(\lambda)$

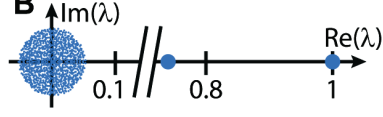

D

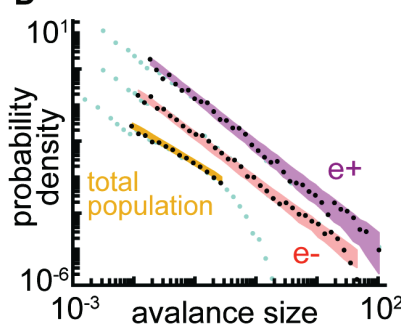

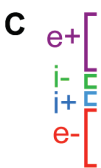
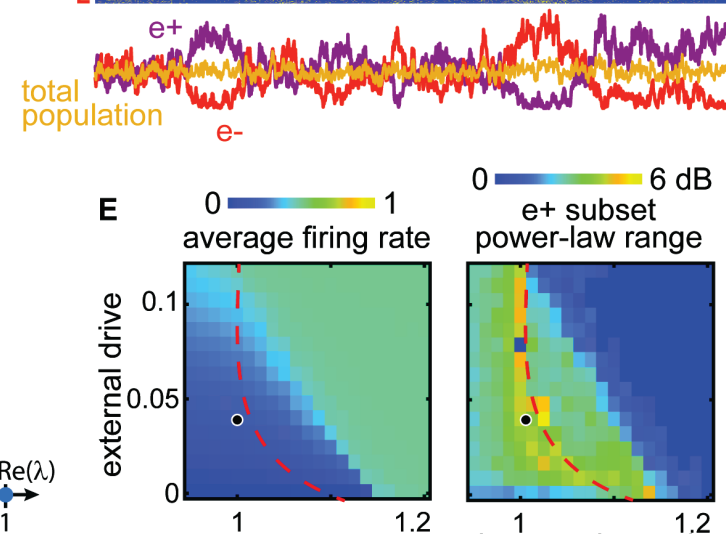

$\mathbf{F}$

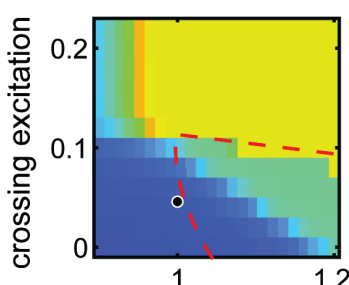

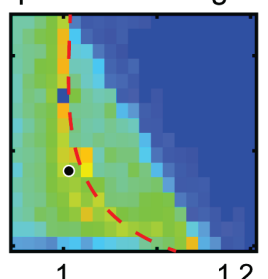

largest eigenvalue of connectivity matrix

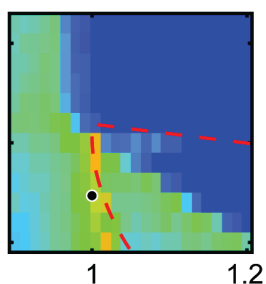

largest eigenvalue of connectivity matrix
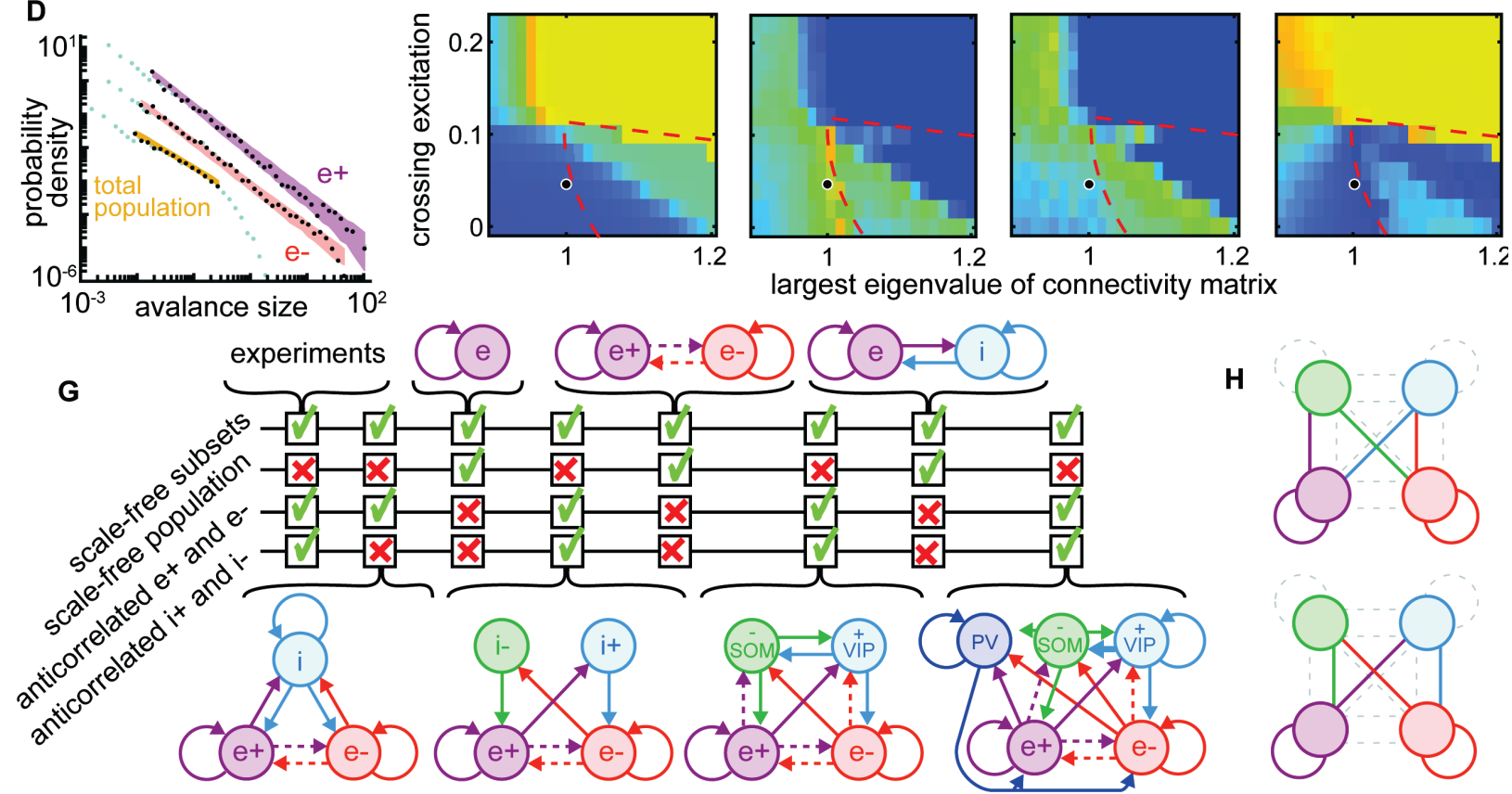

H
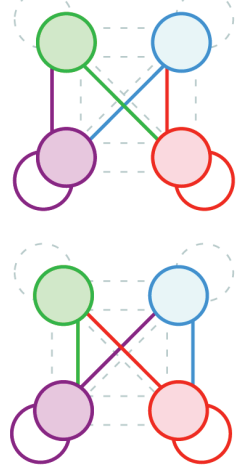

Figure 6. Winner-take-all network structure explains scale-free anticorrelated subsets. A) Connectivity diagram (top) and matrix (bottom) illustrate interactions among four populations. Solid, dashed lines indicate dense $(50 \%)$, sparse (5\%) connectivity, respectively. Two excitatory groups (e+ and e-) inhibit each other via dense crossing inhibition. B) Eigenvalue spectrum of connectivity matrix has two outlying real eigenvalues, the largest equal to 1. C) Timeseries and raster reveal strong anti-correlations between $\mathrm{e}^{+}$and e- populations which cancel out; total population has small fluctuations. D) Neural event distributions for e+ and e- exhibit large power-law range, while the total population does not. E) Parameter space for model. Model agrees with experiments near dashed line for a wide range of input rates, most with largest eigenvalue is near 1. F) Dense crossing excitation from e+ to e- abolishes winner-take-all dynamics. G) Crossing inhibition motif is important for matching our experimental observations. Realistic topologies, including known connectivity among PV+, VIP, and SOM inhibitory neurons (right), contain this crossing inhibition motif and match our observations, but simpler networks can match as well. H) Considering 873000 different network configurations, only 31 match our experimental observations. All matching configurations had one of these two motifs. Dashed lines indicate unnecessary connections.

We found that the e+ and e- neural subsets in this model produced large, slow, scale-free fluctuations (Fig 6C) like those observed in the scale-free subsets of neurons from our experimental observations. The dynamics of e+ were strongly anticorrelated with those of e-, 
and $\mathrm{i}+$ was anticorrelated with $\mathrm{i}$ - (Fig 6C), resulting in cancelation and a lack of scale-free dynamics when the entire population was considered together (Fig 6D). All neurons in the model were driven by unstructured random input; there was no statistical difference between the input to different subsets. Thus, the anticorrelated switching behavior in our model emerges stochastically. These results were robust to a wide range of different input rates (Fig 6E), provided that the largest eigenvalue of the connectivity matrix was near 1 . If the largest eigenvalue was increased beyond 1, the dynamics stopped switching between e+ and e-, instead getting locked into either e+ active and e- inactive or vice versa (this occurred to the right of the red dashed lines in Figs $6 \mathrm{E}$ and F). Thus, the stochastic switching between e+ and e- activity is a type of critical dynamics occurring at a boundary (red dashed line) in the model parameter space. Such critical winner-take-all dynamics never actually "choose a winner," instead switching randomly between the two "equally deserving winners." Scale-free anticorrelated fluctuations were also abolished when the density of connections between $\mathrm{e}+$ and e- were increased beyond about 10\% (Fig 6F). Above this density of connectivity between e+ and e-, the network effectively behaves like a single network, like previous models of criticality and scale-free dynamics.

In addition to testing dependence on the largest eigenvalue, the input rate, and the density of e+to-e- connectivity, we did a systematic exploration of different network structures. We sought to understand if the network structure considered in Fig 6A-F is the only structure that reproduces our experimental observations, or if some other network structures could also work. Fig 6G summarizes the results of testing several other network structures. We found that crossing inhibition mediated by a single inhibitory population could suffice. The disinhibitory motif highlighted by other recent studies (28) is, thus, not necessary to reproduce our observations. Importantly, we showed that our observations can also occur in a more realistic network including a third population of inhibitory neurons with connectivity like that of PV neurons, according approximately to known connectivity $(31,34)$. Finally, we employed a brute force search considering 873,000 different combinations of dense, sparse, or zero connectivity for the 16 connections among the four network nodes in Fig 6A (more detail in Fig S4). We found only 31 configurations that matched the four criteria in Fig 6G. All of these matching configurations included one of two dense crossing inhibition motifs as well as segregated excitatory groups e+ and e- (Fig 6H and Fig S4). In fact, these two motifs were sufficient for predicting which configurations approximately match our experimental results with $80 \%$ accuracy (Methods).

\section{Discussion}

We have shown that ongoing, untrained locomotion, whisking, and pupil diameter changes of mice often exhibit scale-free dynamics. This scale-free behavior is directly related to concurrent scale-free cortical neural activity among certain subsets of neurons, with strong one-to-one correspondence between many behavioral and neural events. What are the functional implications of this close relationship between scale-free behavior and brain activity? In previous work, scale-free neural activity has been associated with multiple functional benefits for sensory information processing $(26,37-39)$ and scale-free behavior has been associated with benefits for foraging, search, and decision making (3-9). Our results suggest that these two lists of functional benefits, previously considered separately, may in fact be a single unified list that occurs together. This longer list of benefits may explain why several types of neural plasticity have been shown to maintain scale-free neural activity $(11,17,37)$.

What mechanisms are responsible for scale-free neural activity? The prevailing view is the criticality hypothesis [see for example $(10,38,40,41)]$. In this view, scale-free dynamics occur 
because the system operates near criticality, i.e. near the tipping point between two distinct dynamical regimes - one synchronous, the other asynchronous. However, previously studied models of criticality do not explain our observations of scale-free activity in limited to subsets of neurons, which were strongly anticorrelated with other subsets of neurons, in apparent stochastic winner-take-all competition. Our model suggests that a new type of criticality must be considered to explain this observation. More specifically, our model suggests that the scale-free dynamics we observe occur at the boundary between winner-less switching and single-winner locked-in dynamics. Additional theoretical efforts are necessary to more fully explore how the traditional criticality hypothesis relates to the competitive criticality suggested by our model.

Setting aside debates about theoretical mechanisms, our results firmly establish that complex fluctuations and events that make up scale-free neural activity are not "background noise" nor "internal cognitive processes" as is often supposed. Our findings call for a revision of these traditional views; scale-free neural activity directly causes (or perhaps is caused by) scale-free behavior and body motion.

\section{References}

1. C. Anteneodo, D. R. Chialvo, Unraveling the fluctuations of animal motor activity. Chaos. 19, 033123 (2009).

2. A. Proekt, J. R. Banavar, A. Maritan, D. W. Pfaff, Scale invariance in the dynamics of spontaneous behavior. Proc. Natl. Acad. Sci. 109, 10564-10569 (2012).

3. D. W. Sims, E. J. Southall, N. E. Humphries, G. C. Hays, C. J. A. Bradshaw, J. W. Pitchford, A. James, M. Z. Ahmed, A. S. Brierley, M. A. Hindell, D. Morritt, M. K. Musyl, D. Righton, E. L. C. Shepard, V. J. Wearmouth, R. P. Wilson, M. J. Witt, J. D. Metcalfe, Scaling laws of marine predator search behaviour. Nature. 451, 1098-1102 (2008).

4. G. M. Viswanathan, S. V. Buldyrev, S. Havlin, M. G. E. Da Luz, E. P. Raposo, H. E. Stanley, Optimizing the success of random searches. Nature. 401, 911-914 (1999).

5. M. E. Wosniack, M. C. Santos, E. P. Raposo, G. M. Viswanathan, M. G. E. da Luz, The evolutionary origins of Lévy walk foraging. PLoS Comput. Biol. 13 (2017), doi:10.1371/journal.pcbi.1005774.

6. K. Garg, C. T. Kello, Efficient Lévy walks in virtual human foraging. Sci. Rep. 11 (2021), doi:10.1038/s41598-021-84542-w.

7. A. Sorribes, B. G. Armendariz, D. Lopez-Pigozzi, C. Murga, G. G. de Polavieja, The origin of behavioral bursts in decision-making circuitry. PLoS Comput. Biol. 7 (2011), doi:10.1371/journal.pcbi.1002075.

8. A. L. Barabási, The origin of bursts and heavy tails in human dynamics. Nature. 435, 207-211 (2005).

9. M. S. Abe, Functional advantages of Lévy walks emerging near a critical point. Proc. Natl. Acad. Sci. U. S. A. 117, 24336-24344 (2020).

10. J. M. Beggs, D. Plenz, Neuronal avalanches in neocortical circuits. J. Neurosci. 23, 11167-77 (2003).

11. W. L. Shew, W. P. Clawson, J. Pobst, Y. Karimipanah, N. C. Wright, R. Wessel, Adaptation to sensory input tunes visual cortex to criticality. Nat. Phys. 11, 659-663 
(2015).

12. G. Scott, E. D. Fagerholm, H. Mutoh, R. Leech, D. J. Sharp, W. L. Shew, T. Knopfel, Voltage Imaging of Waking Mouse Cortex Reveals Emergence of Critical Neuronal Dynamics. J. Neurosci. 34, 16611-16620 (2014).

13. T. Bellay, A. Klaus, S. Seshadri, D. Plenz, Irregular spiking of pyramidal neurons organizes as scale-invariant neuronal avalanches in the awake state. Elife. 4, 1-25 (2015).

14. V. Priesemann, M. Wibral, M. Valderrama, R. Pröpper, M. Le Van Quyen, T. Geisel, J. Triesch, D. Nikolić, M. H. J. Munk, Spike avalanches in vivo suggest a driven, slightly subcritical brain state. Front. Syst. Neurosci. 8, 108 (2014).

15. E. Tagliazucchi, P. Balenzuela, D. Fraiman, D. R. Chialvo, Criticality in large-scale brain FMRI dynamics unveiled by a novel point process analysis. Front. Physiol. 3, 15 (2012).

16. O. Shriki, J. Alstott, F. Carver, T. Holroyd, R. N. A. Henson, M. L. Smith, R. Coppola, E. Bullmore, D. Plenz, Neuronal avalanches in the resting MEG of the human brain. $J$. Neurosci. 33, 7079-90 (2013).

17. Z. Ma, G. G. Turrigiano, R. Wessel, K. B. Hengen, Cortical Circuit Dynamics Are Homeostatically Tuned to Criticality In Vivo. Neuron. 104, 655-664.e4 (2019).

18. S. Yu, T. L. Ribeiro, C. Meisel, S. Chou, A. Mitz, R. Saunders, D. Plenz, Maintained avalanche dynamics during task-induced changes of neuronal activity in nonhuman primates. Elife. 6 (2017), doi:10.7554/eLife.27119.

19. R. Hardstone, S.-S. Poil, G. Schiavone, R. Jansen, V. V Nikulin, H. D. Mansvelder, K. Linkenkaer-Hansen, Detrended fluctuation analysis: a scale-free view on neuronal oscillations. Front. Physiol. 3, 450 (2012).

20. J. M. Palva, A. Zhigalov, J. Hirvonen, O. Korhonen, K. Linkenkaer-Hansen, S. Palva, Neuronal long-range temporal correlations and avalanche dynamics are correlated with behavioral scaling laws. Proc. Natl. Acad. Sci. U. S. A. 110, 3585-90 (2013).

21. C. T. Kello, G. D. a Brown, R. Ferrer-I-Cancho, J. G. Holden, K. Linkenkaer-Hansen, T. Rhodes, G. C. Van Orden, Scaling laws in cognitive sciences. Trends Cogn. Sci. 14, 223232 (2010).

22. H. Yang, W. L. Shew, R. Roy, D. Plenz, Maximal Variability of Phase Synchrony in Cortical Networks with Neuronal Avalanches. J. Neurosci. 32, 1061-1072 (2012).

23. J. Li, W. L. Shew, Tuning network dynamics from criticality to an asynchronous state. PLOS Comput. Biol. 16, e1008268 (2020).

24. J. Wilting, J. Dehning, J. Pinheiro Neto, L. Rudelt, M. Wibral, J. Zierenberg, V. Priesemann, Operating in a reverberating regime enables rapid tuning of network states to task requirements. Front. Syst. Neurosci. 12, 55 (2018).

25. C. Stringer, M. Pachitariu, N. Steinmetz, C. B. Reddy, M. Carandini, K. D. Harris, Spontaneous behaviors drive multidimensional, brainwide activity. Science (80-. ). 364, eaav7893 (2019).

26. S. H. Gautam, T. T. Hoang, K. McClanahan, S. K. Grady, W. L. Shew, Maximizing Sensory Dynamic Range by Tuning the Cortical State to Criticality. PLOS Comput. Biol. 11, e1004576 (2015). 
27. E. Zagha, X. Ge, D. A. McCormick, Competing Neural Ensembles in Motor Cortex Gate Goal-Directed Motor Output. Neuron. 88, 565-577 (2015).

28. P. Garcia-Junco-Clemente, T. Ikrar, E. Tring, X. Xu, D. L. Ringach, J. T. Trachtenberg, An inhibitory pull-push circuit in frontal cortex. Nat. Neurosci. 20, 389-392 (2017).

29. S. Song, P. J. Sjöström, M. Reigl, S. Nelson, D. B. Chklovskii, Highly nonrandom features of synaptic connectivity in local cortical circuits. PLoS Biol. 3, e68 (2005).

30. L. Cossell, M. F. Iacaruso, D. R. Muir, R. Houlton, E. N. Sader, H. Ko, S. B. Hofer, T. D. Mrsic-Flogel, Functional organization of excitatory synaptic strength in primary visual cortex. Nature. 518, 399-403 (2015).

31. X. Jiang, S. Shen, C. R. Cadwell, P. Berens, F. Sinz, A. S. Ecker, S. Patel, A. S. Tolias, Principles of connectivity among morphologically defined cell types in adult neocortex. Science (80-. ). 350, aac9462-aac9462 (2015).

32. N. R. Wall, M. de la Parra, J. M. Sorokin, H. Taniguchi, Z. J. Huang, E. M. Callaway, Brain-wide maps of synaptic input to cortical interneurons. J. Neurosci. 36, 4000-4009 (2016).

33. H.-J. Pi, B. Hangya, D. Kvitsiani, J. I. Sanders, Z. J. Huang, A. Kepecs, Cortical interneurons that specialize in disinhibitory control. Nature. 503, 521-524 (2013).

34. C. K. Pfeffer, M. Xue, M. He, Z. J. Huang, M. Scanziani, Inhibition of inhibition in visual cortex: the logic of connections between molecularly distinct interneurons. Nat. Neurosci. 16, 1068-1076 (2013).

35. O. Kiehn, Decoding the organization of spinal circuits that control locomotion. Nat. Rev. Neurosci. 17 (2016), pp. 224-238.

36. D. B. Larremore, W. L. Shew, J. G. Restrepo, Predicting Criticality and Dynamic Range in Complex Networks: Effects of Topology. Phys. Rev. Lett. 106, 1-4 (2011).

37. W. P. Clawson, N. C. Wright, R. Wessel, W. L. Shew, Adaptation towards scale-free dynamics improves cortical stimulus discrimination at the cost of reduced detection. PLOS Comput. Biol. 13, e1005574 (2017).

38. W. L. Shew, D. Plenz, The functional benefits of criticality in the cortex. Neuroscientist. 19, 88-100 (2013).

39. O. Shriki, D. Yellin, Optimal Information Representation and Criticality in an Adaptive Sensory Recurrent Neuronal Network. PLOS Comput. Biol. 12, e1004698 (2016).

40. M. A. Muñoz, Colloquium: Criticality and dynamical scaling in living systems. Rev. Mod. Phys. 90, 31001 (2018).

41. J. Wilting, V. Priesemann, 25 years of criticality in neuroscience - established results, open controversies, novel concepts. Curr. Opin. Neurobiol. 58, 105-111 (2019).

\section{Acknowledgments:}

Funding: National Institutes of Health grant 1R15NS116742-01 (WLS), National Science Foundation grant 1912352 (WLS), Arkansas Biosciences Institute (WLS). 


\section{Author contributions:}

\section{Conceptualization: WLS}

Data analysis: WLS, SJ, JB

Computational modeling: WLS, JB

Funding acquisition: WLS

$$
\text { Writing: WLS, SJ, JB }
$$

Competing interests: Authors declare that they have no competing interests.

Data and materials availability: The data analyzed here were first published in Stringer et al. (25) and are publicly available at doi: 10.25378/janelia.6163622.v4. Analysis code is publicly available at doi: TBD

\section{Supplementary Materials}

Materials and Methods

Figs. S1 to S4 


\title{
Supplementary Materials for
}

\section{Scale-free behavioral dynamics directly linked with scale-free cortical dynamics}

10

\author{
Sabrina Jones ${ }^{1}$, Jacob Barfield ${ }^{1}$, Woodrow L Shew ${ }^{1 *}$ \\ Correspondence to: $\underline{\text { shew@uark.edu }}$
}

This PDF file includes:

15

Materials and Methods

Figs. S1 to S4 


\section{Materials and Methods}

Animals and data acquisition

The data analyzed here were first published in Stringer et al. (25) and are publicly available at doi: 10.25378/janelia.6163622.v4. All animal protocols and data acquisition methods are described in the original publication. Here we analyzed the data from multiplane calcium imaging during periods with no visual stimulation. Our labeling of the different recordings corresponds to the original authors' labeling system of the data as follows:

mouse 1 - spont_M150824_MP019_2016-04-05, mouse 2 - spont_M160825_MP027_2016-12-12, mouse 3 - spont_M160907_MP028_2016-09-26, mouse 4a-spont_M161025_MP030_2016-11-20, mouse 4b-spont_M161025_MP030_2017-06-16, mouse 4c - spont_M161025_MP030_2017-06-23, mouse 5 - spont_M170714_MP032_2017-08-04, mouse 6 - spont_M170717_MP033_2017-08-18, mouse 7 - spont_M170717_MP034_2017-08-25.

Neural data pre-processing - Beginning from the deconvolved fluorescence traces (variable called Fsp in the original data set), first, the data were z-scored. That is, for each neuron, we subtracted its time-averaged activity and divided by its standard deviation. Second, we applied a low-pass filter (cutoff frequency $0.2 \mathrm{~Hz}, 2$ nd order butterworth filter, using Matlab filtfilt function).

\section{$25 \quad$ Power-law fitting and range}

To assess the scale-free-ness of behavioral events and neural population events, we developed an algorithm for finding the range of event sizes that are well fit by a power law. The algorithm is based on a maximum likelihood fitting procedure as established in previous [e.g. $(11,36)]$. We fit the measured event size distribution with a truncated power-law with minimum event size $s_{\text {min }}$ and maximum event size $s_{\max }$, excluding data that fell outside these bounds during the fit process. Similar to previous studies, our algorithm has two fitting parameters - $s_{\min }$ and the power-law exponent. Smax was not a fitting parameter; it was chosen to be the largest observed event size. We tested exponents between 0.7 and 2 in steps of 0.02 . We tested $s_{\min }$ values between the smallest observed event size and the largest observed event size, increasing in 10 logarithmically spaced increments per decade. Thus, the power-law range reported in the manuscript has a resolution of $0.1 \mathrm{~dB}$. Importantly, our fitting algorithm is entirely independent of any choice of binning that might be used to visualize the event size distribution.

For our purposes of measuring power law range, we needed to improve upon previously reported algorithms, our algorithm accounted for (excluded) confounding outlier event sizes. These "outliers" were rare events that caused noise in the extremes of the distribution tail or head and, in some cases, caused spuriously large power-law range estimates. We defined outliers by first ranking all event sizes from smallest to largest. Next, we computed differences in sizes (in $\mathrm{dB}$ ) for consecutive sizes in this ranked list. The outliers have a large difference in size compared to the following size (or preceding size). We define outliers as events with a size difference greater than $3 \%$ of the total range in $\mathrm{dB}$. We also tried $6 \%$ as a defining outlier cutoff and found that our results were not very sensitive to this choice (Fig $1 \mathrm{C}$ ). 
The fitting algorithm executed the following steps. First, outliers were excluded. Second, events with size less than Smin were excluded. Third, the maximum likelihood power-law exponent was calculated. Fourth, we assessed the goodness-of-fit. We repeated these four steps for all the possible $s_{\min }$ values, in the end, identifying the largest range (smallest $s_{\min }$ ) that passed our goodness-of-fit criterion.

The goodness-of-fit criterion we adopted here was also new, to our knowledge, and better suited to our goals of assessing power-law range, compared to previous methods. The steps for quantifying goodness-of-fit were as follows. First, we created a cumulative distribution function (CDF) of the real data. Second, we created 500 surrogate data sets drawn from the best-fit truncated power-law. Third, we created $500 \mathrm{CDFs}$, one for each of the surrogate data sets. Fourth, we resampled all 501 CDFs with 10 logarithmically space points per decade, linearly interpolated. Fifth, we calculated the fraction $\mathrm{F}$ of points in the resampled CDF of the real data that fell within the bounds of the 500 resampled surrogate CDFs. We explored various goodness-of-fit criteria between $\mathrm{F}=0.75$ and $\mathrm{F}=0.9$ for the behavioral data (Fig 1C) and the neural data (Fig 2E). This is a rather conservative goodness-of-fit test, much more conservative than the more typically used Kolmogorov-Smirnov statistic for example.

\section{Statistical significance of correlations}

Fig 4 of the main manuscript was based on computing correlation coefficients of short time series of behavior and neural activity. The number of such correlation coefficients computed for each behavioral event was 1000, because there were 1000 neural subsets. In the manuscript we concluded that a significant number of these correlations were strong. This conclusion was arrived at by comparing to time-shifted control neural data (same as described for the timeshifted controls in the manuscript for Fig 2). For each behavioral event, we obtained 1000 timeshifted control correlation coefficients. We then counted how many of the real correlation coefficients were greater than 999 of the time-shifted control correlation coefficients. By chance, we should expect this count to be 1 . We concluded that there was a significant number of strong correlations if this count was greater than 4. A similar reasoning was used to conclude that there were a significant number of strongly correlated behavioral events for each neural subset.

\section{Computational model}

The model consisted of $\mathrm{N}=1000$ binary neurons; the state of the ith neuron at time $\mathrm{t}$ is $s_{i}(t)=0$ (quiescent) or $s_{i}(t)=1$ (firing). The population was divided into 4 groups called $\mathrm{e}+, \mathrm{e}-, \mathrm{i}+$, and $\mathrm{i}-$, as diagramed in Fig 6A. The e+ and e- groups include 400 excitatory neurons each; $i+$ and $i-$ include 100 inhibitory neurons each. The dynamics of the neurons are updated synchronously according to

$$
s_{i}(t)= \begin{cases}1 & \text { with probability } p_{i}(t) \\ 0 & \text { with probability } 1-p_{i}(t)\end{cases}
$$

where the activation probability is

$$
p_{i}(t)=f\left(\eta+\sum_{j=1}^{N} C_{i j} s_{j}(t-1)\right) .
$$

Here, $\eta$ is a constant representing input from outside the model network. We characterized how changes in $\eta$ affect model dynamics in Fig 6E (vertical axis, labeled "external drive"). The connection from neuron $\mathrm{j}$ to neuron $\mathrm{i}$ is given by $C_{i j}$, one element of the connection matrix 
depicted in Fig 6B. Certain pairs of groups were sparsely connected, while other pairs of groups were densely connected, or not connected at all. 50\% of connections (randomly chosen) were zero for two densely connected groups. 95\% of connections (randomly chosen) were zero for a sparsely connected pair of groups. All non-zero excitatory connections were set to the same constant $c$; all inhibitory connections were set to $-c$. The value of $c$ was set by first normalizing the entire connection matrix by its largest eigenvalue and then multiplying by another constant $\Lambda$ to obtain the desired largest eigenvalue. We studied how the model dynamics depend on $\Lambda$ in Fig 6F and Fig 6G (horizontal axis). For the shotgun search of many different circuit configurations, we set $\Lambda=1$. We also studied how the density of connectivity between e+ and e- impacted model dynamics in Fig 6F (vertical axis, called "crossing excitation").

All model data analysis was done on the population activity, averaged over neurons for each group.

For the shotgun search for model configurations that result in dynamics consistent with our experimental results, we first generated a list of all possible configurations of the 16 pairwise group connections. We considered three possible densities for each of these connections: disconnected $(0 \%)$, sparse $(5 \%)$, or dense $(50 \%)$. Thus, the list of all possible connection configurations includes $3^{16}$ (more than 43 million) possibilities. Clearly testing all these possibilities would take a long time, so we pared down the list with the following constraints, which exclude several very unrealistic possibilities:

1. Both $\mathrm{e}+$ and e- must have non-zero within-group connectivity

2. No disconnected components were allowed. Every node had to be reachable by at least one connection.

3. There must be at least one excitatory to inhibitory connection

4. There must be at least one inhibitory to excitatory connection After applying these constraints the list of possible configurations still included 18576000 possible circuits. We tested 873000 of these possibilities, chosen at random. We considered a circuit to be consistent with experimental results if it met the following conditions:

1. Correlation coefficient of e+ and e- must be less than -0.5

2. Correlation coefficient of $i+$ and $i-$ must be less than -0.5

3. Power law range of e+ or e- must be greater than $3.5 \mathrm{~dB}$

4. Power law range of the total population must be less than $2 \mathrm{~dB}$

We found that only 31 circuit configurations met these conditions. These matching networks are illustrated in Fig S4. As noted in the main text, all of the matching configurations featured two structural motifs. First, they included dense self-connections within e+ and within e-, but weak or non-existent connections between $\mathrm{e}+$ and e-. Second, all of the matching configurations included one of two dense crossing inhibition configurations (Fig 6H). Moreover, we found that these two conditions were sufficient to predict which networks would match the experimental results with $80 \%$ accuracy, if we loosened the criteria for matching experiments slightly. These looser criteria were:

1. Correlation coefficient of e+ and e- must be less than -0.5

2. Correlation coefficient of $i+$ and $i-$ must be less than -0.2

3. Power law range of e+ or e- must be at least 1.4 times that of the total population 


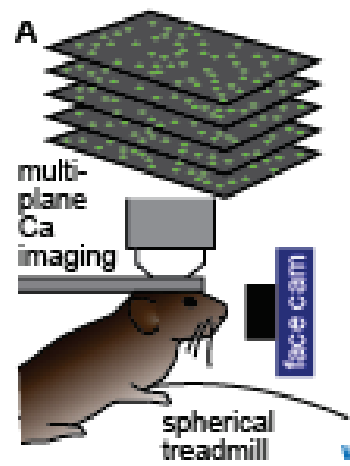

B
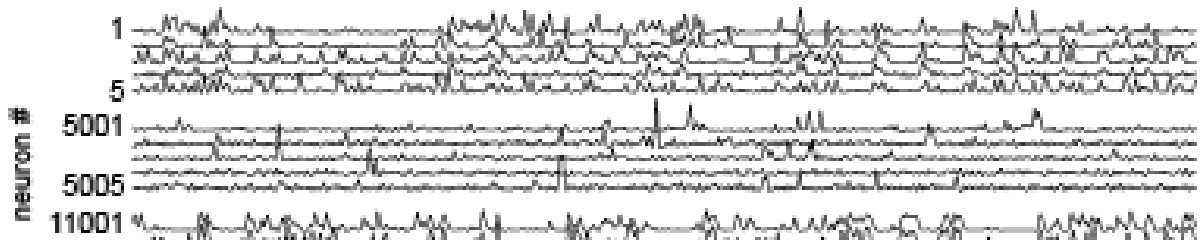

당

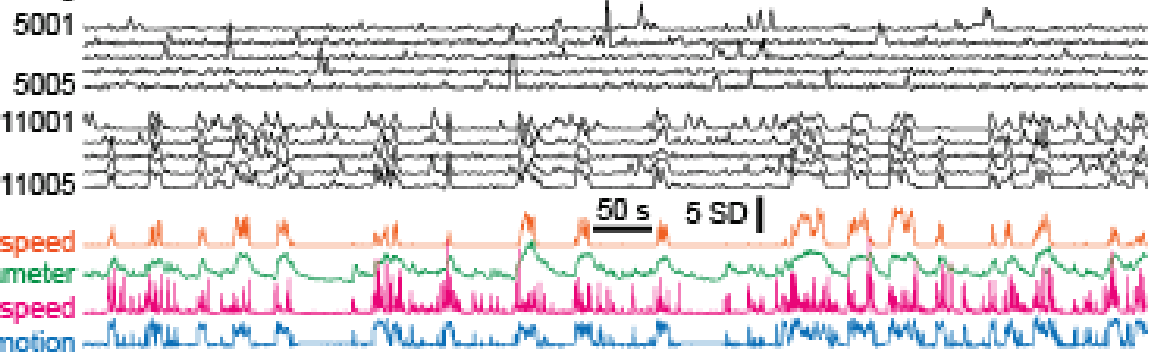

Figure S1: Simultaneous recording of behavior and neural activity. A) Multiplane calcium imaging measured 10000 neurons in mouse visual cortex, while a camera measured facial motion and a treadmill measured run speed. B) Example activity time series from 15 neurons (out of 11005 total in this recording). Notice some neurons were strongly correlated with behavior (bottom 5), while others were anticorrelated (top 5), and others were nearly uncorrelated (middle 5). C) The four aspects of behavior - run speed, pupil diameter, speed of changes in gaze direction, and whisker motion - tended to covary. 

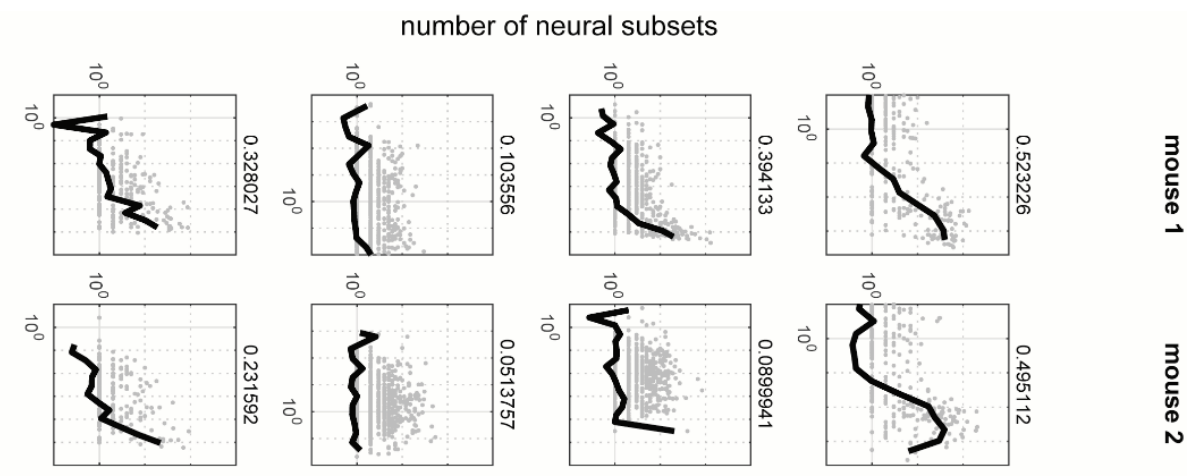

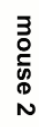
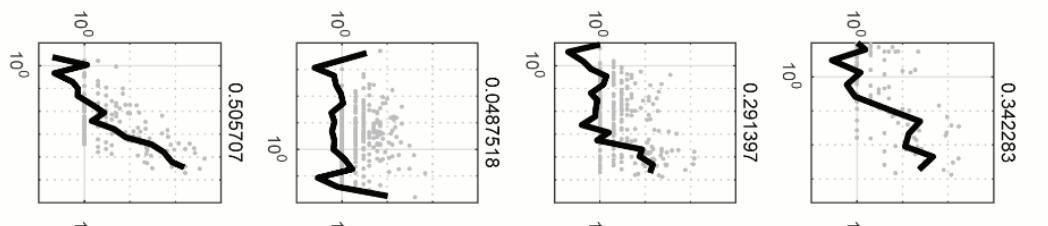

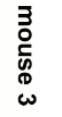
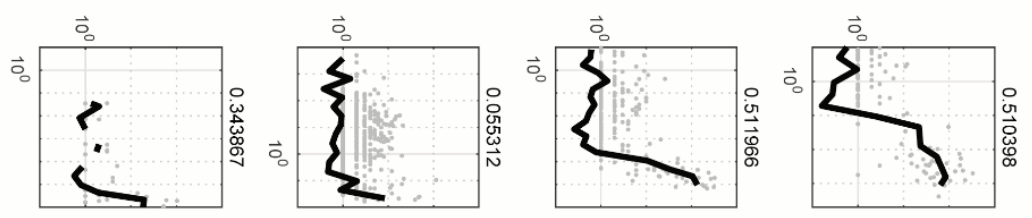

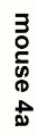
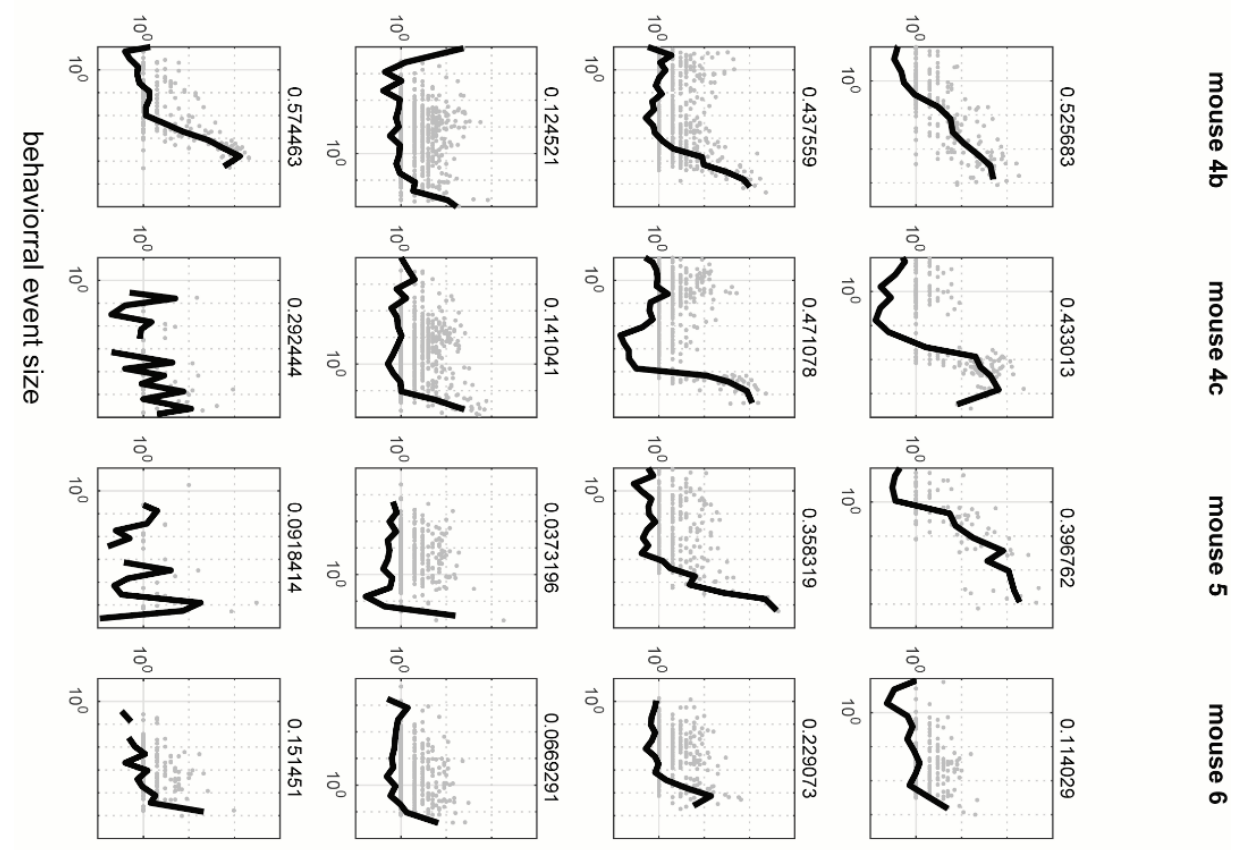

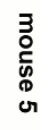
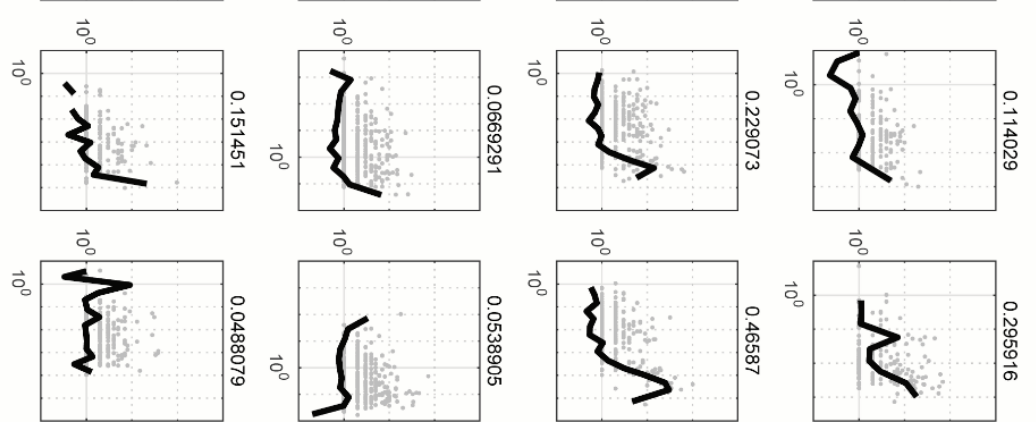

3
อ
$\frac{0}{0}$
0
0
0

Figure S2: These plots present the same results as Fig 4B, but for all mice and all behaviors. The left column represents pupil diameter changes, second column represents gaze dynamics, third column represents whisking, right column represents running. The number on the right side of each plot represents the fraction shown in Fig 4D. 


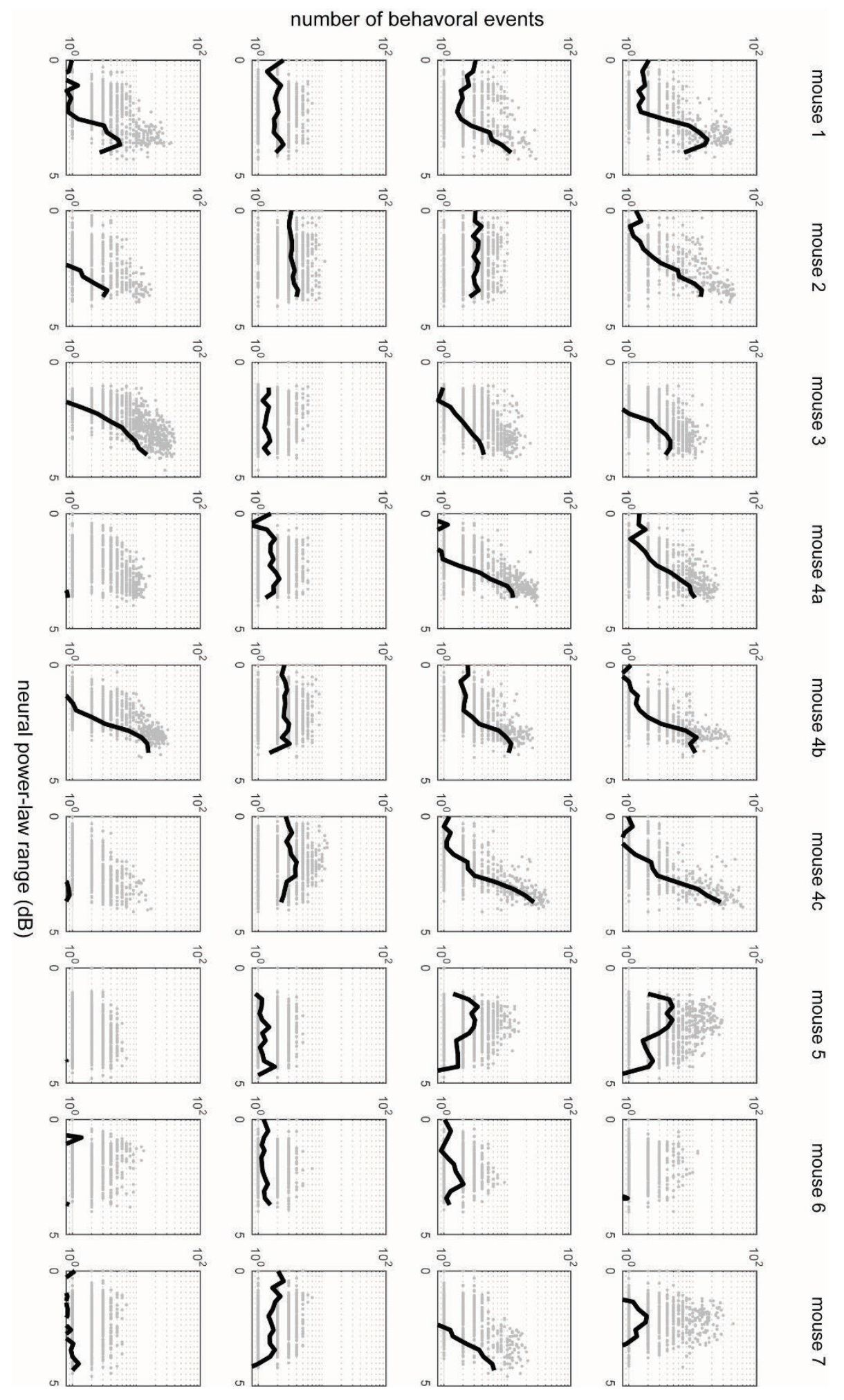

Figure S3: These plots present the same results as Fig 4C, but for all mice and all behaviors. The left column represents pupil diameter changes, second column represents gaze dynamics, third column represents whisking, right column represents running. 

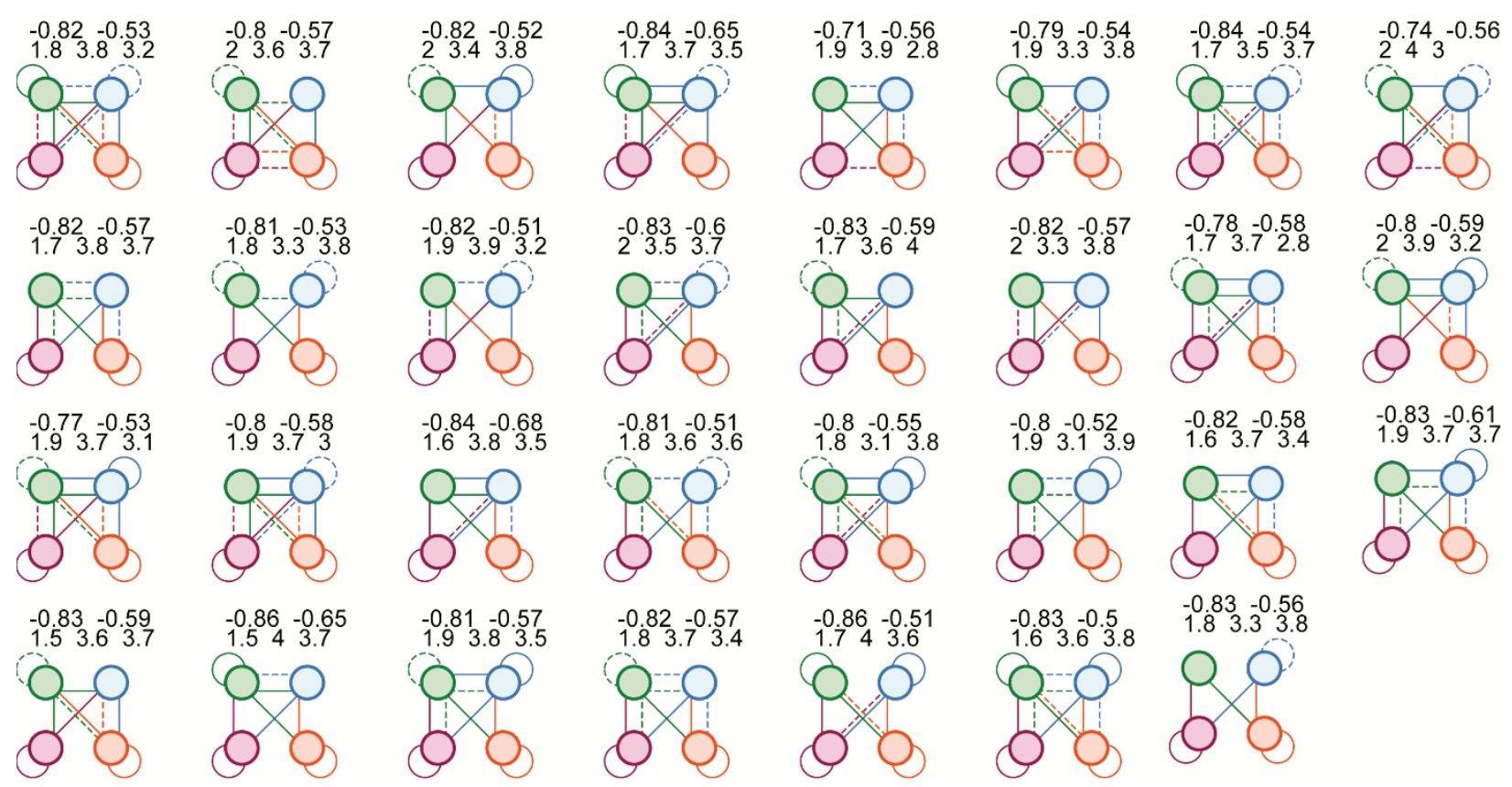

Figure S4: We tried 873000 different network configurations. Here we show the 31 configurations that successfully reproduced our primary experimental results. The color scheme and meaning of solid and dashed lines is the same as in Fig 6. The numbers above each circuit diagram report the correlation coefficient between e+ and e- (top left), the correlation coefficient between $\mathrm{i}+$ and $\mathrm{i}$ - (top right), power-law range for total population (bottom left), power-law range for e+ (bottom middle), and power-law range for e- (bottom right). 